\title{
Bak is a key molecule in apoptosis induced by methanol extracts of Codonopsis lanceolata and Tricholoma matsutake in HSC-2 human oral cancer cells
}

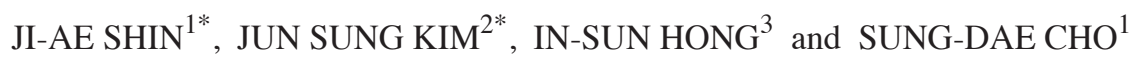 \\ ${ }^{1}$ Department of Oral Pathology, School of Dentistry, Institute of Oral Bioscience, Chonbuk National University, \\ Jeonju; ${ }^{2}$ R\&D Center, Biterials Co. Ltd.; ${ }^{3}$ Department of Veterinary Public Health, \\ Laboratory of Stem Cell and Tumor Biology, Seoul National University, Seoul, Republic of Korea
}

Received June 13, 2012; Accepted August 30, 2012

DOI: $10.3892 / \mathrm{ol} .2012 .898$

\begin{abstract}
Since the 5-year survival rate of oral cancer remains low, more effective and non-toxic therapeutic and preventive strategies are required. Certain natural products possess anticancer properties. The present study investigated the effects of the methanol extracts of Codonopsis lanceolata (MECI) and Tricholoma matsutake (METM) and identified the molecular target in HSC-2 human oral cancer cells. The results revealed that MECI and METM inhibited growth and induced apoptosis, as demonstrated by poly (ADP-ribose) polymerase (PARP) cleavage and nuclear condensation and fragmentation. The compounds also increased Bak protein expression, while Bax, Bcl-XL and Mcl-1 were not affected. The results of the present study show that MECI and METM induce apoptosis to inhibit tumor growth of HSC-2 cells by modulating the Bak protein and suggest that Codonopsis lanceolata and Tricholoma matsutake are potential anticancer drug candidates for oral cancer.
\end{abstract}

\section{Introduction}

Over the last several decades, numerous studies have demonstrated that a number of naturally occurring compounds exhibit beneficial effects for human health, including cancer chemopreventive properties (1-3). Despite the improvement of oral cancer treatment with conventional strategies, including surgery, radiotherapy and chemotherapy in recent years, the 5 -year mortality rate remains low at approximately $50 \%(4,5)$. For this reason, cancer biologists have focused on the use of natural products as an alternative therapeutic tool to improve the survival rate of oral cancer patients.

Correspondence to: Professor Sung-Dae Cho, Department of Oral Pathology, School of Dentistry and Institute of Oral Bioscience, Chonbuk National University, Jeonju 561-756, Republic of Korea E-mail: efiwdsc@chonbuk.ac.kr

Key words: Bak, Codonopsis lanceolata, Tricholoma matsutake, apoptosis, oral cancer
Codonopsis lanceolata is a perennial herb that grows naturally in moist places in woodlands and hills and is commonly found in East Asia (6). Codonopsis lanceolata has been used as a traditional medicine in Korea, Japan and China $(7,8)$. The plant has been demonstrated to have anti-inflammatory properties and a protective effect against ischemic damage and alcoholic fatty liver $(9,10)$. Recently, Codonopsis lanceolata has been demonstrated to affect apoptosis and cell cycle arrest in colon cancer and leukemia cells, therefore this herb is likely to also have therapeutic potential against oral cancer $(11,12)$. Tricholoma matsutake is an ectomycorrhizal fungus that is economically significant in Japan (13). As a traditional edible fungus in oriental countries, it has been used as a vegetable and a traditional Chinese medicine for the prevention and treatment of disease for several thousand years (14). It has been shown that a novel polysaccharide isolated from Tricholoma matsutake has antioxidant (14) and immunostimulatory properties (15). However, the anticancer activities of these two natural products require further investigation.

The mitochondrial apoptotic signaling pathway is mainly governed by $\mathrm{Bcl}-2$ protein family members. The Bcl-2 family consists of anti- and pro-apoptotic proteins. The anti-apoptotic proteins, including Bcl-2, Bcl-XL and Mcl-1, contain four Bcl-2 homology $(\mathrm{BH})$ domains, whereas the pro-apoptotic members are divided into proteins with three BH domains (e.g., Bax, Bak and Bok) and BH3-only proteins (e.g., Bim, Bad and Bik). Bak, one of the multi-domain proapoptotic proteins, is essential for apoptotic cell death (16). Bak-deficient Jurkat T leukemia cells were resistant to apoptosis triggered by ultraviolet or anticancer drugs and the expression of antisense Bak in breast cancer cells increased the resistance to cisplatin-induced apoptosis $(17,18)$. Thus, Bak is markedly implicated in apoptotic cell death in various types of cancer cells.

The present study aimed to determine whether Codonopsis lanceolata and Tricholoma matsutake possess anticancer properties in HSC-2 human oral cancer cells. The results of this study reveal that two natural products induce apoptosis to inhibit the growth of oral cancer cells by regulating the Bak protein. 


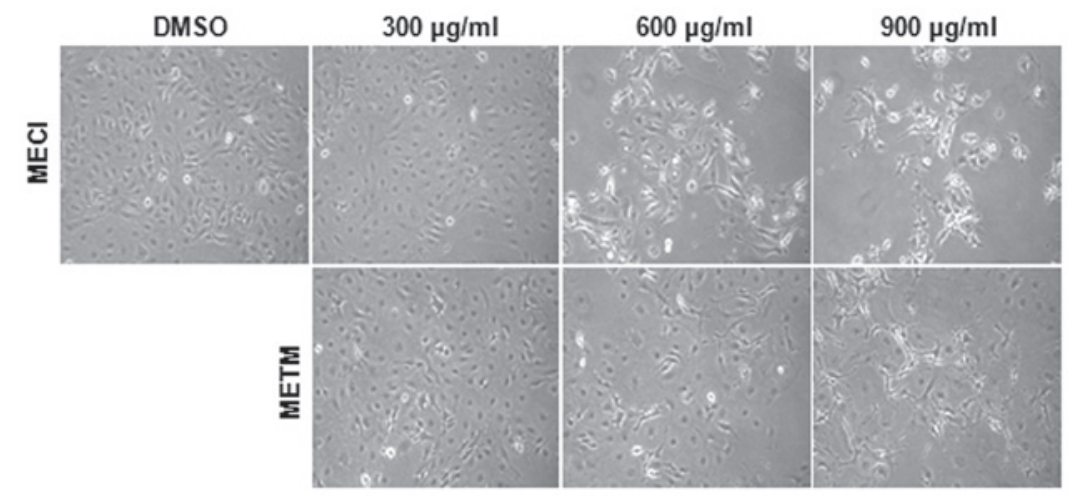

Figure 1. Effects of MECI and METM on morphological changes in HSC-2 cells. HSC-2 cells were treated with DMSO or various doses of MECI and METM $(300,600$ and $900 \mu \mathrm{g} / \mathrm{ml})$ for $48 \mathrm{~h}$. The morphological changes of HSC-2 cells by MECI and METM were observed and photographed at identical magnifications. MECI, methanol extracts of Codonopsis lanceolata; METM, methanol extracts of Tricholoma matsutake.

The study was approved by the ethics committee of Chonbuk National University (Chonbuk National University, Jeon-ju, Korea).

\section{Materials and methods}

Chemicals and antibodies. MECI and METM were provided by Professor Ki-Han Kwon (Kwangju University, Kwangju, Korea). Poly (ADP-ribose) polymerase (PARP) antibody was purchased from BD Pharmingen ${ }^{\mathrm{TM}}$ (San Jose, CA, USA). Antibodies against Bak, Bax, Bcl-xL and Mcl-1 were obtained from Cell Signaling Technology (Charlottesville, VA, USA). Actin antibody was supplied from Santa Cruz Biotechnology Inc. (Santa Cruz, CA, USA).

Cell culture and treatments. HSC-2 oral squamous carcinoma cells were provided by Hokkaido University (Hokkaido, Japan). Cells were cultured in DMEM supplemented with $10 \%$ FBS and antibiotics at $37^{\circ} \mathrm{C}$ in a $5 \% \mathrm{CO}_{2}$ incubator. Cells were treated with DMSO or various doses of MECI and METM (300, 600 and $900 \mu \mathrm{g} / \mathrm{ml}$ ) for 24 or $48 \mathrm{~h}$.

3-(4,5-dimethylthiazol-20yl)-(3-carboxymethoxyphenyl)-2-(4sulphophenyl)-2H-tetrazolium (MTS) assay. The effects of MECI and METM on cell growth were examined by the CellTiter 96R Aqueous One Solution Cell Proliferation Assay kit (Promega, Madison, WI, USA). HSC-2 cells were seeded in 96-well plates and then treated with DMSO or various doses of MECI and METM $(300,600$ and $900 \mu \mathrm{g} / \mathrm{ml})$ for 24 and $48 \mathrm{~h}$. MTS solution was added to each well and incubated for $2 \mathrm{~h}$ at $37^{\circ} \mathrm{C}$. The absorbance was analyzed using a ELISA microplate reader (Bio-Tek Instruments, Inc., Madison, WI, USA) at 490 and $690 \mathrm{~nm}$ (background).

Western blot analysis. HSC-2 cells were seeded and treated with DMSO or various doses of MECI and METM (300, 600 and $900 \mu \mathrm{g} / \mathrm{ml}$ ) for $48 \mathrm{~h}$. Cells were harvested and then whole cell lysates were extracted with lysis buffer. The protein concentrations were analyzed by DC Protein Assay (Bio-Rad Laboratories, Hercules, CA, USA). Samples containing equal amounts of protein were separated by SDS-PAGE and then transferred to Immun-Blot ${ }^{\mathrm{TM}}$ PVDF membranes (Bio-Rad Laboratories). The membranes were blocked with 5\% skimmed milk in TBST at room temperature for $1 \mathrm{~h} 30 \mathrm{~min}$ and incubated overnight at $4^{\circ} \mathrm{C}$ with primary antibodies against PARP, Bak, Bax, Bcl-xL, Mcl-1 and actin, followed by incubation with HRP-conjugated secondary antibodies at room temperature for $1 \mathrm{~h} 30 \mathrm{~min}$. The membranes were detected by ECL Western Blotting Luminol reagent (Santa Cruz Biotechnology, Inc.).

DAPI staining. The effects of MECI and METM on chromatin condensation and nuclear fragmentation were detected using a fluorescent nuclear dye, DAPI (4'-6-diamidino-2-phenylindole; Sigma, St. Louis, MO, USA). HSC-2 cells were seeded and treated with DMSO or various doses $(300,600$ and $900 \mu \mathrm{g} / \mathrm{ml})$ of MECI and METM. After $48 \mathrm{~h}$, cells were harvested by trypsinization and resuspended in PBS. The cells were fixed in $100 \%$ methanol at room temperature (RT) for $10 \mathrm{~min}$ and then deposited on slides and stained with DAPI solution $(2 \mu \mathrm{g} / \mathrm{ml})$. DAPI-stained cells were observed under a fluorescence microscope equipped with a suitable filter for the DAPI fluorescent dye.

\section{Results}

MECI and METM significantly decrease the growth of HSC-2 cells. The effects of MECI and METM on cell viability were first investigated. The morphological changes of HSC-2 cells were observed with a microscope following treatment with various doses of MECI and METM $(300,600$ and $900 \mu \mathrm{g} / \mathrm{ml})$ for $48 \mathrm{~h}$. The results revealed that cells treated with MECI and METM were detached from plates and floated with rounded shapes. Marked morphological changes of HSC-2 cells were observed following higher doses of MECI and METM (Fig. 1). The effect of MECI and METM on cell viability was investigated using an MTS assay. As shown in Fig. 2A and B, MECI and METM significantly decreased cell viability in a dose- and time-dependent manner. The calculated $\mathrm{IC}_{50}$ values of MECI and METM were 692.57 and $685.87 \mu \mathrm{g} / \mathrm{ml}$ at $48 \mathrm{~h}$, respectively. These results indicate that the treatment of HSC-2 cells with MECI and METM inhibits tumor cell growth.

MECI and METM markedly induce apoptosis in HSC-2 cells. To demonstrate whether the growth-inhibitory effects of MECI and METM were due to apoptosis, a western blot 

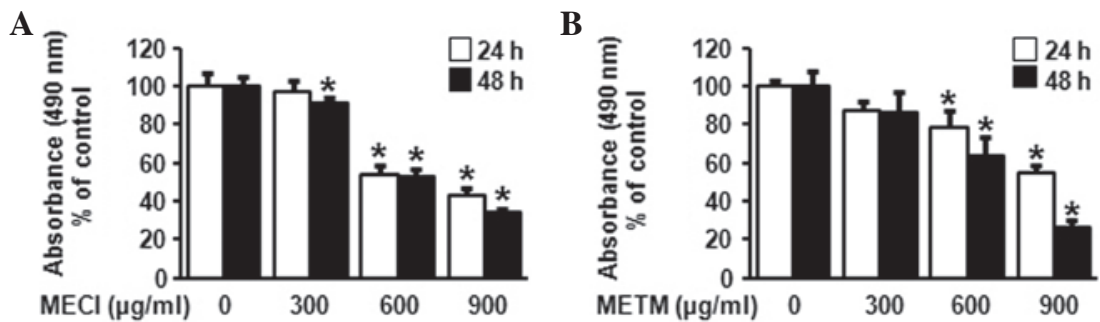

Figure 2. Growth inhibitory effects of MECI and METM in HSC-2 cells. HSC-2 cells were treated with DMSO or various doses of (A) MECI and (B) METM $(300,600$ and $900 \mu \mathrm{g} / \mathrm{ml})$ for 24 and $48 \mathrm{~h}$. The cell viability was evaluated using the MTS assay and expressed in graphs as mean \pm SD. ${ }^{*} \mathrm{P}<0.05$ compared with the DMSO-treated group. MECI, methanol extracts of Codonopsis lanceolata; METM, methanol extracts of Tricholoma matsutake; MTS, 3-(4,5-dimethylthiazol-20yl)-(3-carboxymethoxyphenyl)-2-(4-sulphophenyl)-2H-tetrazolium.
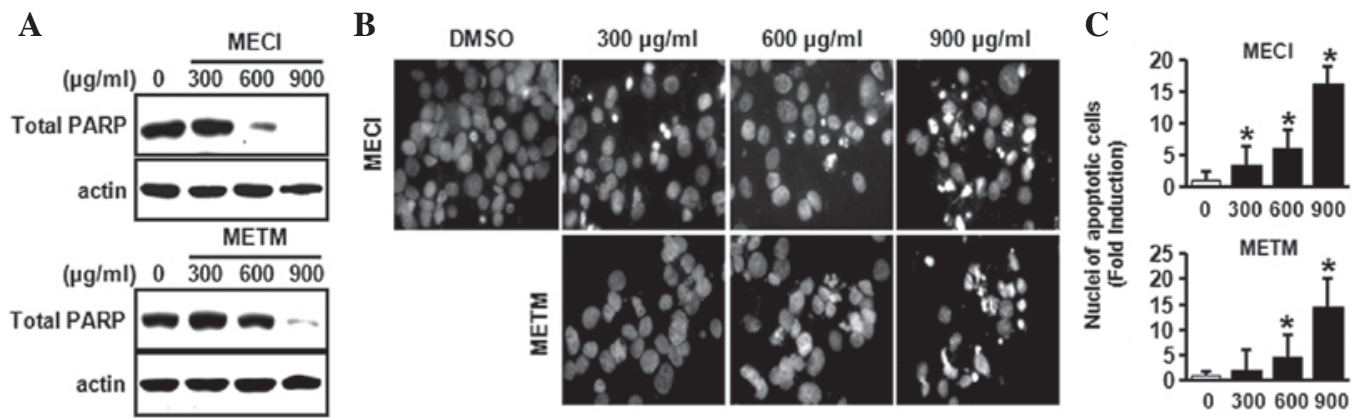

Figure 3. Apoptotic effects of MECI and METM in HSC-2 cells. HSC-2 cells were treated with DMSO or various doses of MECI and METM (300, 600 and $900 \mu \mathrm{g} / \mathrm{ml}$ ) for $48 \mathrm{~h}$. (A) The effects of MECI and METM on PARP protein were analyzed by western blot analysis. (B) The effects of MECI and METM on nuclear condensation and fragmentation were detected by DAPI staining using the fluorescence microscope. Magnification, $\mathrm{x} 400$. (C) The number of apoptotic cells presented as mean $\pm \mathrm{SD} .{ }^{*} \mathrm{P}<0.05$ compared with the DMSO-treated group. MECI, methanol extracts of Codonopsis lanceolata; METM, methanol extracts of Tricholoma matsutake; PARP, poly (ADP-ribose) polymerase.

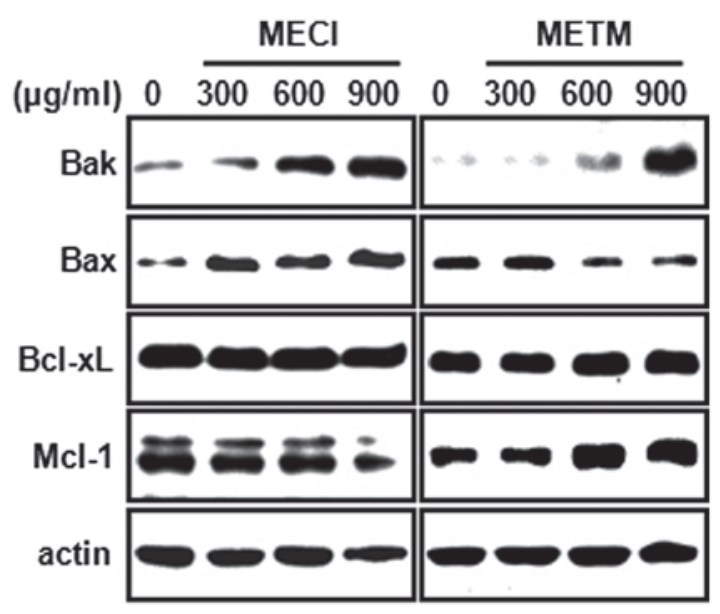

Figure 4. Effects of MECI and METM on expression of Bcl-2 family proteins. HSC-2 cells were treated with DMSO or various doses of MECI and $\operatorname{METM}(300,600$ and $900 \mu \mathrm{g} / \mathrm{ml})$ for $48 \mathrm{~h}$. The expression of Bcl-2 family proteins was detected by western blot analysis using antibodies against Bak, Bax, Bcl-xL and Mcl-1. MECI, methanol extracts of Codonopsis lanceolata; METM, methanol extracts of Tricholoma matsutake.

analysis was performed using the PARP antibody and DAPI staining. As shown in Fig. 3A, MECI and METM markedly decreased total PARP expression, indicating that MECI and METM may increase cleaved PARP. Furthermore, the effects of MECI and METM on apoptosis were also confirmed by DAPI staining. Fig. 3B and C indicate that MECI and METM significantly increased the number of DAPI-stained apoptotic cells, as demonstrated by nuclear condensation and fragmentation. These results suggest that MECI and METM induce apoptosis in HSC-2 oral cancer cells.

MECI and METM increase Bak expression in HSC-2 cells. In order to further explore the molecular mechanisms of MECI- and METM-induced apoptosis, the apoptotic effects of MECI and METM in HSC-2 cells with regard to pro- or anti-apoptotic proteins in mitochondria were studied. As shown in Fig. 4, MECI and METM increased the expression of the pro-apoptotic protein Bak in a dose-dependent manner. However, the pro-apoptotic protein Bax was changed only by MECI. By contrast, MECI and METM had no significant effect on the anti-apoptotic proteins Bcl-xL and Mcl-1. These results reveal that the induction of Bak protein by MECI and METM may be commonly associated with MECI- and METM-induced apoptosis.

\section{Discussion}

Cancer chemoprevention includes the use of natural synthetic or biological compounds to prevent cancer development. Numerous studies have demonstrated that natural products play critical roles against oral cancer. For example, green tea extracts (GTEs) reduce the incidence of oral cancer development by inhibiting invasion and metastasis $(19,20)$. Withania somnifera extract also reduces the overall occurrence of oral tumors by increasing antioxidant activity $(21,22)$. 
Recently, it has been demonstrated that Polygonum cuspidatum induces apoptotic cell death in oral cancer cells through the regulation of specificity protein 1 (23). This clearly suggests that the extracts of natural products have anticarcinogenic activities against oral cancer. Codonopsis lanceolata extract induces apoptosis in human colon tumor HT-29 cells and human acute promyelocytic leukemia HL-60 cells, while Tricholoma matsutake has also exhibited antitumor effects $(11,12,24)$, indicating that these two natural products may have anticarcinogenic properties. However, no study concerning their anticancer effects against oral cancer has been published. Thus, the present study investigated the anticancer effects of MECI and METM. The results demonstrated that MECI and METM significantly decreased cell viability and induced apoptosis, consistent with the results of previous studies.

Permeabilization of the mitochondria during apoptosis is a critical control point for the regulation of programmed cell death (25). Thus, it is important to investigate the Bcl-2 family protein that regulates the integrity of the mitochondria. Numerous studies have shown that natural products regulate the mitochondrial membrane potential to induce apoptosis in cancer cells. Anonaine, an alkaloid compound extracted from the leaves of Michelia alba, induced apoptosis through Bax- and caspase-dependent pathways in human cervical cancer (HeLa) cells (26). Verticinone, a major alkaloid isolated from the bulbus of Fritillaria ussuriensis, also induced apoptosis by damaging mitochondrial membrane potential (increase of Bax/decrease of Bcl-2) in immortalized and malignant human oral keratinocytes (27). Conversely, certain natural products have protective effects against apoptosis. Ginkgo biloba extract protected rat pheochromocytoma (PC12) cells from possible oxidative damage induced by trophic factors and garlic extracts are also protective against the apoptosis of intestinal epithelial cells caused by methotrexate $(28,29)$. Therefore, the effect of natural products on mitochondrial function remains controversial. The current study also investigated whether MECI and METM affect Bcl-2 family proteins, including Bak, Bax, Bcl-XL and Mcl-1. The results demonstrated that only Bax was affected by MECI and METM in HSC-2 cells. This suggests that MECI- and METM-induced apoptosis is associated with the regulation of Bak protein and that the extracts damage mitochondrial membrane potential instead of protecting it. Neise et al (30) reported that Mcl-7 human breast cancer cells expressing Bak protein were more sensitive to apoptosis induced by staurosporine and TRAIL. Activation of Bak also increased the sensitivity of cisplatin-induced apoptosis in melanoma cell lines (31). These results support the theory that Bak is a significant molecule in MECI- and METM-induced apoptosis. The homo-oligomerization of Bak is thought to be responsible for mitochondrial outer membrane permeabilization, therefore it is likely to be investigated in future studies.

In conclusion, our results suggest that MECI and METM inhibit tumor growth of HSC-2 cell lines and induce apoptosis. This effect is due to the induction of Bak protein. Therefore, this study presents Codonopsis lanceolata and Tricholoma matsutake as potential anticancer drug candidates targeting Bak in oral cancer.

\section{Acknowledgements}

This study was supported by the Basic Science Research Program through the National Research Foundation of Korea (NRF) funded by the Ministry of Education, Science and Technology (2012002481 and 2012003731).

\section{References}

1. Tsuda $\mathrm{H}$, Ohshima $\mathrm{Y}$, Nomoto $\mathrm{H}$, et al: Cancer prevention by natural compounds. Drug Metab Pharmacokinet 19: 245-263, 2004.

2. Nobili S, Lippi D, Witort E, et al: Natural compounds for cancer treatment and prevention. Pharmacol Res 59: 365-378, 2009.

3. Kuno T, Tsukamoto T, Hara A and Tanaka T: Cancer chemoprevention through the induction of apoptosis by natural compounds. Biophys Chem 3: 156-173, 2012.

4. Nagler RM: Saliva as a tool for oral cancer diagnosis and prognosis. Oral Oncol 45: 1006-1010, 2009.

5. Rahman MA, Amin AR and Shin DM: Chemopreventive potential of natural compounds in head and neck cancer. Nutr Cancer 62: 973-987, 2010

6. Guo WL, Gong L, Ding ZF, et al: Genomic instability in phenotypically normal regenerants of medicinal plant Codonopsis lanceolata Benth. et Hook. f., as revealed by ISSR and RAPD markers. Plant Cell Rep 25: 896-906, 2006.

7. Lee KT, Choi J, Jung WT, Nam JH, Jung HJ and Park HJ: Structure of a new echinocystic acid bisdesmoside isolated from Codonopsis lanceolata roots and the cytotoxic activity of prosapogenins. J Agric Food Chem 50: 4190-4193, 2002.

8. Lee YG, Kim JY, Lee JY, et al: Regulatory effects of Codonopsis lanceolata on macrophage-mediated immune responses. J Ethnopharmacol 112: 180-188, 2007.

9. Cho K, Kim SJ, Park SH, Kim S and Park T: Protective effect of Codonopsis lanceolata root extract against alcoholic fatty liver in the rat. J Med Food 12: 1293-1301, 2009.

10. Byeon SE, Choi WS, Hong EK, et al: Inhibitory effect of saponin fraction from Codonopsis lanceolata on immune cell-mediated inflammatory responses. Arch Pharm Res 32: 813-822, 2009.

11. Wang L, Xu ML, Hu JH, Rasmussen SK and Wang MH: Codonopsis lanceolata extract induces G0/G1 arrest and apoptosis in human colon tumor HT-29 cells - involvement of ROS generation and polyamine depletion. Food Chem Toxicol 49: 149-154, 2011.

12. Lee KW, Jung HJ, Park HJ, Kim DG, Lee JY and Lee KT: Beta-D-xylopyranosyl-(1-->3)-beta-D-glucuronopyranosylechinocystic acid isolated from the roots of Codonopsis lanceolata induces caspase-dependent apoptosis in human acute promyelocytic leukemia HL-60 cells. Biol Pharm Bull 28: 854-859, 2005.

13. Lian C, Narimatsu M, Nara K and Hogetsu T: Tricholoma matsutake in a natural Pinus densiflora forest: correspondence between above- and below-ground genets, association with multiple host trees and alteration of existing ectomycorrhizal communities. New Phytol 171: 825-836, 2006.

14. Ding X, Tang J, Cao M, et al: Structure elucidation and antioxidant activity of a novel polysaccharide isolated from Tricholoma matsutake. Int J Biol Macromol 47: 271-275, 2010.

15. Kim JY, Byeon SE, Lee YG, et al: Immunostimulatory activities of polysaccharides from liquid culture of pine-mushroom Tricholoma matsutake. J Microbiol Biotechnol 18: 95-103, 2008.

16. Wei MC, Zong WX, Cheng EH, et al: Proapoptotic BAX and BAK: a requisite gateway to mitochondrial dysfunction and death. Science 292: 727-730, 2001.

17. Hemmati PG, Gillissen B, von Haefen C, et al: Adenovirusmediated overexpression of p14(ARF) induces p53 and Bax-independent apoptosis. Oncogene 21: 3149-3161, 2002.

18. Wang GQ, Gastman BR, Wieckowski E, et al: A role for mitochondrial Bak in apoptotic response to anticancer drugs. J Biol Chem 276: 34307-34317, 2001.

19. Ho YC, Yang SF, Peng CY, Chou MY and Chang YC: Epigallocatechin-3-gallate inhibits the invasion of human oral cancer cells and decreases the productions of matrix metalloproteinases and urokinase-plasminogen activator. J Oral Pathol Med 36: 588-593, 2007. 
20. Chiang WC, Wong YK, Lin SC, Chang KW and Liu CJ: Increase of MMP-13 expression in multi-stage oral carcinogenesis and epigallocatechin-3-gallate suppress MMP-13 expression. Oral Dis 12: 27-33, 2006

21. Manoharan S, Panjamurthy K, Balakrishnan S, Vasudevan $\mathrm{K}$ and Vellaichamy L: Circadian time-dependent chemopreventive potential of withaferin-A in 7,12-dimethylbenz[a] anthracene-induced oral carcinogenesis. Pharmacol Rep 61: 719-726, 2009.

22. Panjamurthy K, Manoharan S, Nirmal MR and Vellaichamy L: Protective role of Withaferin-A on immunoexpression of p53 and bcl-2 in 7,12-dimethylbenz(a)anthracene-induced experimental oral carcinogenesis. Invest New Drugs 27: 447-452, 2009.

23. Shin JA, Shim JH, Jeon JG, et al: Apoptotic effect of Polygonum cuspidatum in oral cancer cells through the regulation of specificity protein 1 . Oral Dis 17: 162-170, 2011.

24. Ebina T: Antitumor effects of intratumoral injection of Basidiomycetes preparations. Gan To Kagaku Ryoho 32: 1654-1656, 2005 (In Japanese).

25. Danial NN and Korsmeyer SJ: Cell death: critical control points. Cell 116: 205-219, 2004.
26. Chen CY, Liu TZ, Tseng WC, Lu FJ, Hung RP and Chen $\mathrm{CH}$ : (-)-Anonaine induces apoptosis through Bax- and caspase-dependent pathways in human cervical cancer (HeLa) cells. Food Chem Toxicol 46: 2694-2702, 2008.

27. Yun YG, Jeon BH, Lee JH, et al: Verticinone induces cell cycle arrest and apoptosis in immortalized and malignant human oral keratinocytes. Phytother Res 22: 416-423, 2008.

28. Li T, Ito K, Sumi S, Fuwa T and Horie T: Protective effect of aged garlic extract (AGE) on the apoptosis of intestinal epithelial cells caused by methotrexate. Cancer Chemother Pharmacol 63: 873-880, 2009.

29. Shi C, Yao Z, Xu J and Yew DT: Effects of Gingko Extract (EGb761) on oxidative damage under different conditions of serum supply. J Bioenerg Biomembr 41: 61-69, 2009.

30. Neise D, Graupner V, Gillissen BF, et al: Activation of the mitochondrial death pathway is commonly mediated by a preferential engagement of Bak. Oncogene 27: 1387-1396, 2008.

31. Mandic A, Viktorsson K, Molin M, et al: Cisplatin induces the proapoptotic conformation of Bak in a deltaMEKK1-dependent manner. Mol Cell Biol 21: 3684-3691, 2001 